\title{
COVID-19: The impacts on foundation training in district general hospitals in the East of England and the East Midlands
}

\author{
Author: Archana Sasitharan ${ }^{\mathrm{A}}$
}

The COVID-19 pandemic has impacted the training of foundation doctors across the UK. A survey of foundation year 1 doctors across several district general hospitals in the East of England and East Midlands deaneries was carried out to investigate their perceptions of the impact on their training.

KEYWORDS: COVID-19, FY1, foundation, training, learning

DOI: $10.7861 /$ clinmed.2020-0592

\section{Background}

The COVID-19 pandemic has altered what we define as 'the norm' in many aspects of our lives, including the way in which healthcare is delivered. In the UK, those in training programmes have found themselves redeployed across departments, to areas requiring additional support in providing high quality of care for unwell patients and meeting the demands that a pandemic brings. Many have found themselves stepping outside their comfort zones, brushing up on medical knowledge that they may not have touched for years since specialising. Those who have only recently graduated from medical school have found themselves in a situation last observed in 1918, while others have expedited their journey as qualified professionals, taking up posts as foundation interim year 1 doctors.

With the halt in training across most specialty programmes within the UK, including the foundation training programme, many did not rotate across specialties over the April period, during which the numbers of COVID-19 cases and fatalities were rapidly increasing. Health Education England (HEE) advised that foundation year 1 (FY1) doctors should not rotate, and those in community placements should be brought back to hospital to meet the demands at the front door. ${ }^{1}$ Teaching programmes were halted, with concerns over how to maintain social distancing and observe infection control procedures while safely disseminating knowledge relevant to the climate FY1 doctors found themselves in. In the early phase of the pandemic, no formal online platform had been identified to provide teaching on a regular basis.

Author: Afoundation year 1 doctor, Broomfield Hospital, Chelmsford, UK
Furthermore, cancellations in clinics and operations meant specialties relying heavily on elective appointments had stopped functioning at full capacity, thus limiting learning opportunities. The UK Foundation Programme Office (UKFPO), which issues guidance for FY1 doctors on their training, released information on changes to portfolios and how doctors would be assessed at the end of the academic year, as well as providing e-learning resources for continued professional development. ${ }^{2}$

Following the restructuring of services, each trainee has had their training impacted in various ways. A qualitative online survey was disseminated to FY1 doctors across several district general hospitals in the East of England and East Midlands deaneries to gain further insight on this matter.

\section{Survey results}

49 responses were received from participants working in a range of specialties across general medicine and surgery. Only one participant was in a community placement. 45 (91.8\%) participants did not rotate onto their third job, with 29 (59.2\%) doctors being re-deployed to other fields. 13 (26.5\%) participants had a change in career aspirations following working through the pandemic. Only 32 (65.3\%) of those who participated had worked in both a medical and surgical specialty during their FY1 training. $17(34.7 \%)$ of those who filled in the survey would be working as an $\mathrm{SHO}$ in either a medical or surgical specialty despite not having worked in it as an FY1, with some suggesting they may not be as prepared to take on increased responsibility due to unfamiliarity with the practical application of their knowledge. Interestingly, only $13(26.5 \%)$ doctors received formal teaching throughout the pandemic, yet the majority $(89.8 \%)$ would have preferred formal teaching to have continued. 27 (55.1\%) participants felt opportunities for further progression (such as research and audit projects, operating time in theatre, attending clinics and clerking) had been lost during this period.

$30(61.2 \%)$ participants felt that rotating onto their third job would have benefitted them in regard to their training, with the remaining trainees stating a number of reasons why they disagreed. Those who said no reasoned that logistics and patient safety during a pandemic should take priority over their training. Remaining in the same specialty would mean that doctors were familiar with their routine and hence could continue safely without having to be introduced to a new department and a new team. Those who felt they should have rotated raised a number 
of points. Several doctors working in surgical specialties felt that rotating onto a surgical job would not have been beneficial for learning, as the majority of cases were cancelled, and departments were functioning on a skeleton basis. One doctor mentioned that their reason for changing career aspirations was encouraged by working in the same field for 8 months.

A few felt that it may have been appropriate to rotate in June when the number of patients presenting with COVID-19 had reduced, giving further exposure in other fields. Some felt that familiarisation with a new job and new environment would have only taken a few weeks, whereas others wished to rotate so that they would have experience in both medical and surgical specialties in preparation for foundation year 2 (FY2) training, where they would be senior house officers (SHOs) and taking referrals. Several doctors stated they had chosen a specific set of jobs as it contained a specialty they wished to pursue in the future, and then had missed out on experiencing it. A few doctors mentioned that as the most junior members of the team, they would have had very little to contribute to the management of COVID-19, and hence they should have been allowed to continue with their training, while more senior trainees should have remained.

\section{Discussion}

How may COVID-19 impact future decisions that the current FY1 cohort make? Those who may have chosen a certain specialty with the aim of building their applications for specialty training may have to take time out after foundation training to seek further training opportunities. A number of doctors may feel they have become deskilled through limited learning opportunities at present.

Adaptations may need to be made over the upcoming year. For example, trainees can be directed to relevant and specific e-learning modules, or using remote teaching platforms such as Zoom or Microsoft Teams to restart weekly teaching programmes. Online seminars hosted by specialty doctors may allow FY1 doctors to explore fields they have not had the opportunity to work in. Simulation sessions can be created in small groups, focusing on the transition between FY1 and SHO. Opportunities to shadow SHOs during on-call periods could be created, similar to the 'Preparation for practice' programme that has been rolled out in the UK for those starting as FY1 doctors. Those rotating onto Foundation Year 2 of training should be given built-in opportunities to do 'taster weeks' in a specialty they want to explore, particularly if they did not get to rotate onto it in FY1. Barriers to organising this would include clashes in rotas and identifying supervisors to guide the trainee accordingly. However, this may be particularly useful in encouraging those considering specialties such as general practice, where there is minimal exposure during FY1 training but which are in need of doctors.

There are a number of other factors that need to be taken into account. Each hospital took an independent route in determining how to reallocate resources and utilise their staff to cope with demand, including changing rotas and shift patterns, which would have influenced how each FY1 doctor was impacted. Those who were changed on to the emergency 'COVID rota', which consisted of alternating 12-hour day and night shifts, may have had skewed perceptions of a career in hospital medicine due to the nature of the rota, whereas others may have become attracted to the idea of it. Further questions identifying specifically how career aspirations may have changed and why, and how each doctor felt they could be better supported during the remainder of their training could be identified.

A similar independent qualitative study was conducted in the North Wales region, ${ }^{3}$ where some comparisons could be made. A stark difference was observed with the number of foundation trainees re-deployed, with only $6.9 \%$ of participants transferred to other specialties in North Wales compared to $59.2 \%$ within East of England and East Midlands regions. This may have reflected differences in hospital pressures, with England persistently having more cases of COVID-19 than Wales. Both studies have identified that the majority of participants felt that the loss of opportunity of rotating onto their third specialties, combined with the reduction in teaching opportunities, have left trainees unprepared for further progression. However, a larger proportion of participants from the survey conducted in North Wales voiced this concern, suggesting regions with fewer demands may not have had to disrupt all training programmes for the full duration of the pandemic. It would also have been interesting to compare data areas such as Central London, where the impact of the COVID-19 pandemic may have been greater.

All in all, the uniqueness of the situation we have found ourselves in in the twenty-first century has proven devastating, but a lot has been learnt. It has altered the way we perceive and practise medicine and has impacted us in many ways beyond our career. As the dust starts to settle, it's important to re-establish what has been lost and adjust to a new way of learning and training.

\section{Acknowledgements}

The author would like to acknowledge her fellow colleagues who participated in the survey.

\section{References}

1 Health Education England. Coronavirus (COVID-19) Information for Trainees. HEE, 2020. www.hee.nhs.uk/coronavirus-information-trainees [Accessed 14 July 2020].

2 UK Foundation Programme. Impact of COVID-19 on the UK Foundation Programme 2020. UKFPO, 2020. https://foundationprogramme.nhs.uk/covid-19/ [Accessed 14 July 2020].

3 Prince S, Adhiyaman V. What was the impact of COVID-19 on the foundation training programme in North Wales? Future Health J 2020, in press (doi: 10.7861/fhj.2020-0026).

Address for correspondence: Dr Archana Sasitharan, Broomfield Hospital, Mid and South Essex NHS Foundation Trust, Court Road, Broomfield, Chelmsford CM1 7ET, UK. Email: archana-s@doctors.org.uk 